\title{
Dimorfismo sexual de la leptina, resistencia a la insulina y composición corporal en prepúberes normopeso
}

\author{
Leptin sexual dimorphism, insulin resistance, and body composition in normal weight \\ prepubescent
}

\author{
Jenny Vitery R.a,d, Guillermo Ortega ${ }^{\mathrm{b}, \mathrm{d}}$, Blanca C. Salazar C. ${ }^{\mathrm{c}, \mathrm{d}}$
}

aEscuela de Rehabilitación Humana, Facultad de Salud, Universidad del Valle. Facultad de Salud de la Universidad Santiago de Cali. Cali, Colombia 'Departamento de Ciencias Básicas de la Salud, Facultad de Ciencias de la Salud, Pontificia Universidad Javeriana. Cali, Colombia 'Departamento de Ciencias Fisiológicas. Facultad de Salud, Universidad del Valle. Cali, Colombia ¿Grupo Nutrición, Facultad de Salud, Universidad del Valle. Cali, Colombia

Recibido: 5 de septiembre de 2019; Aceptado: 10 de septiembre de 2020

¿Qué se sabe del tema que trata este estudio?

La etapa prepuberal es un periodo crítico del desarrollo de la grasa corporal, en el cual la leptina y la resistencia a la insulina han sido asociados, sin embargo, hay pocos estudios en prepúberes normopeso.
¿Qué aporta este estudio a lo ya conocido?

En prepúberes normopeso se confirman diferencias por sexo en adiposidad y en niveles de leptina, no asociadas con diferencias en el IMC ni con la resistencia a la insulina.

\section{Resumen}

La etapa prepuberal es un periodo crítico del desarrollo de la grasa corporal, en el cual la leptina y la resistencia a la insulina han sido asociados, sin embargo, hay pocos estudios en prepúberes normopeso. Objetivo: Evaluar la relación de leptina con composición corporal y resistencia a la insulina en un grupo de prepúberes normopeso. Pacientes y Método: Estudio transversal analítico en 128 prepúberes saludables, normopeso, entre 6 y 10 años. Se midieron, talla, peso, índice de masa corporal (IMC), porcentaje de grasa corporal (PGC), perímetro abdominal (PA) y perímetro de cadera (PC). Se determinó leptina $(\mathrm{ng} / \mathrm{ml})$ e Insulina $(\mu \mathrm{U} / \mathrm{L})$ en plasma por inmunoensayo y glicemia $(\mathrm{mmol} / \mathrm{L})$ por método enzimático. Se calculó HOMA-IR. Se hizo análisis de comparación y de correlación por sexo. Resultados: Se confirmó en niñas un mayor nivel de leptina $(6,8 \pm 5$ vs 3,3 $\pm 3,7 ; \mathrm{p}=0,000)$, insulina $(7,1 \pm 4,5$ vs $5,2 \pm 2,5 ; \mathrm{p}=0,016)$, PGC $(22,4 \pm 4,3$ vs $18,6 \pm 3,9 ; \mathrm{p}=0,000)$ y PC $(67 \pm 5,7$ vs $65,0 \pm 4,5 ; \mathrm{p}=0,019)$, y un menor índice cintura/cadera $(0,84 \pm 0,04$ vs $0,88 \pm 0,04 ; \mathrm{p}=0,000)$ comparado con varones. Las correlaciones de leptina con variables antropométricas fueron significantes en ambos sexos, con mayor asociación en sexo femenino. La asociación del HOMA-IR con
Palabras clave:

Leptina;

Resistencia a la

insulina;

Porcentaje de grasa;

Prepúberes;

Sexo 
la leptina fue similar en ambos sexos. Conclusiones: En prepúberes normopeso de 6 a 10 años, hay diferencias por sexo en adiposidad y en niveles de leptina, no asociadas con diferencias en el IMC ni en la resistencia a la insulina. La mayor asociación de leptina con adiposidad en las niñas podría estar relacionada con una elevada tasa de adipogénesis inducida por esta hormona.
Keywords: Leptin; Insulin resistance; Percentage of fat; Prepubescents; Sex

\section{Introducción}

Durante la etapa prepuberal, alrededor de los 6 años, ocurre el rebote adiposo (RA). El momento de inicio de este rebote puede definirse como la edad en que se produce el incremento del Índice de masa corporal (IMC) luego de alcanzar su valor más bajo. Este incremento del IMC se atribuye fundamentalmente a la ganancia de tejido graso ${ }^{1,2}$ que ocurre entre los 6 a los 10 años, periodo en el cual, la concentración de las hormonas sexuales son incipientes ${ }^{3}$ por lo que su efecto no es determinante en este etapa. El RA antes de los 5,5 años o un IMC mayor en la etapa prepuberal han sido asociados con obesidad en la adultez $z^{2,4,5}$. La ganancia de grasa en los prepúberes ocurre predominantemente por adipogénesis ${ }^{3,6}$. Uno de los factores asociados a este proceso es la leptina, hormona peptídica producida principalmente por los adipocitos subcutáneos, la que ha sido descrita como marcador de adipogénesis ${ }^{7}$. Dado que una de sus funciones es disminuir la saciedad a nivel hipotalámico y aumentar el gasto calórico periférico, su disfunción ha sido asociada a obesidad ${ }^{7}$ y a resistencia a la insulina en el adulto ${ }^{3}$. Además, el aumento del tamaño del adipocito se ha relacionado con el incremento de su concentración y esta se correlaciona con la masa grasa total ${ }^{3,8}$. La leptina ha sido estudiada principalmente en prepúberes con sobrepeso y obesidad ${ }^{9,10}$, condiciones en las que predomina la hipertrofia de adipocitos. En los prepúberes normopeso, en los que la adipogénesis es determinante ${ }^{2}$, el dimorfismo por sexo de la concentración de leptina y su relación con resistencia a la insulina y composición corporal ha sido poco explorado ${ }^{11,12}$.

El objetivo de este estudio fue evaluar, por sexo, la relación entre la concentración de leptina circulante con la composición corporal y la resistencia a la insulina en un grupo de prepúberes normopeso.

\section{Pacientes y Método}

Este es un estudio transversal analítico realizado en niños y niñas prepúberes, escolarizados de la ciudad de Cali-Colombia, entre octubre de 2017 y marzo de 2018. Para calcular el tamaño de muestra se asumió un poder del $80 \%$ y un tamaño del efecto de la leptina por edad de 0,115 , estimando un $\mathrm{n}=80$ sujetos, que garantizó la relación funcional entre las variables del estudio, sin embargo, al final se reclutaron 128 prepúberes mejorando la potencia a un 95\%. Se consideró como peso normal aquel cuyo IMC que se encontraba entre -1DS $\mathrm{y}+1 \mathrm{DS}^{13}$.

Para identificar a los niños y niñas normopeso se midieron y pesaron 1.076 escolares aparentemente saludables, con edades entre 6 a 10 años de seis colegios de educación básica primaria, pertenecientes a un es- 
trato socioeconómico medio. Aunque se seleccionaron 345 , tuvieron un peso normal 128 sujetos (62 niños y 66 niñas) con un estado Tanner I (pre puberal), diagnosticado por valoración médica ${ }^{14,15}$. Se excluyeron los sujetos con tratamiento de control del peso, con deficiencias endocrinas, neurológicas, con procesos inflamatorios diagnosticados mediante Proteína $\mathrm{C}$ reactiva ultrasensible (PCR-Us) en plasma $(>0,43 \mathrm{mg} / \mathrm{L})^{4}$, o que de manera voluntaria se retiraran del estudio. Los padres y los niños seleccionados aceptaron y firmaron el consentimiento y el asentimiento informado respectivamente.

Este estudio fue aprobado por el Comité de Ética Humana de la Universidad del Valle - Colombia (acta No. 005-017), según las disposiciones de la declaración de Helsinki y de la normatividad de las instituciones educativas participantes.

\section{Caracterización sociodemográfica y mediciones antropométricas}

Mediante encuesta se obtuvo información sociodemográfica. La medición antropométrica fue realizada en los 128 sujetos de acuerdo a las directrices del ISAK (International Society for the Advancement of Kinanthropometry $)^{16}$. Las medidas antropométricas se obtuvieron con los sujetos en ropa ligera, sin calzado y luego de vaciamiento vesical. Para el peso $(\mathrm{kg})$ se utilizó una báscula mecánica (SECA automática, modelo 761 de esfera, $\pm 100 \mathrm{~g}$ ), con un estadiómetro de pared (SECA $240 \pm 1 \mathrm{~mm}$ ), se midió la talla $(\mathrm{cm})$. Con estas mediciones se calculó el IMC $\left(\mathrm{kg} / \mathrm{m}^{2}\right)$. Para medir el perímetro abdominal $(\mathrm{cm})$ y el perímetro de cadera $(\mathrm{cm})$, se usó cinta métrica de acero flexible e inextensible (Harpenden $\pm 0,1 \mathrm{~cm}$ ). El perímetro abdominal (PA) se midió al final de la espiración, colocando la cinta métrica en el punto medio entre el borde inferior de la décima costilla y la cresta ilíaca. El perímetro de la cadera (PC) se midió colocando la cinta métrica alrededor de la cadera a nivel del máximo relieve de los músculos glúteos y la sínfisis pubiana en posición bípeda y los pies juntos ${ }^{16}$.

El porcentaje de grasa corporal (PGC) se obtuvo con la medición de los pliegues cutáneos tríceps y subescapular con un plicómetro (Harpenden $\pm 0,2 \mathrm{~mm}$, con presión constante de $\left.10 \mathrm{~g} / \mathrm{mm}^{2}\right)^{17}$. El PGC se estimó con la ecuación de Slaughter ${ }^{18}$. El error técnico asociado a la medición fue de 2,5\%, inferior al aceptado por esta ecuación.

\section{Determinaciones bioquímicas}

Las concentraciones plasmáticas de leptina, insulina, glucosa y PCR-Us, se midieron en muestras de sangre obtenidas por venopunción de 110 sujetos, luego de un ayuno de 12 horas. El plasma obtenido por centrifugación fue almacenado a $-20^{\circ} \mathrm{C}$. Para la medi- ción de insulina por inmunoensayo (Architect Insulin Reagent Kit, Abbott Laboratories) se usaron $24 \mu \mathrm{L}$ de plasma con una sensibilidad del kit $<1,0 \mu \mathrm{U} / \mathrm{mL}^{19}$. En la determinación de la leptina (Leptin ELISA LDN-kit) se utilizaron $20 \mu \mathrm{L}$ de plasma por duplicado, con un límite de detección de $0,5 \mathrm{ng} / \mathrm{mL}^{20}$. La glicemia se determinó por la técnica de la glucosa oxidasa (Glucose, Abbot, Clinical Chemistry, USA) utilizando $2 \mu \mathrm{L}$ de muestra y la determinación de la PCR-Us se hizo por turbidimetría (Multigen CRP Vario ${ }^{\circledR}$; Abbott, Wiesbaden, Germany) con $2 \mu \mathrm{L}$ de muestra con un límite de detección de $0,1 \mathrm{mg} / \mathrm{L}$. El grado de resistencia a la insulina (RI) se determinó por el índice HOMA-IR, calculado como: HOMA-IR $=$ insulina $(\mu \mathrm{U} / \mathrm{mL}) \times$ glucosa $(\mathrm{mmol} / \mathrm{L})] / 22,5^{21}$.

\section{Análisis estadístico}

La descripción según el sexo de las variables continuas: edad, composición corporal (talla, peso, IMC, PGC, PA, PC, ICC) y de las variables bioquímicas (glicemia, insulina, HOMA-IR), se realizó a través de la media y la mediana como medidas de tendencia central y se utilizaron como medidas de dispersión, la desviación estándar ( $\pm \mathrm{DE})$ o el intervalo de confianza (IC) del 95\%. La distribución normal fue evaluada con el test de ShapiroWilk. Para el análisis de comparaciones, las variables que no se ajustaron a una distribución normal fueron evaluadas por el test de la U de Mann-Whitney y en el caso contrario, por el test de Student. Las correlaciones bivariadas entre las variables antropométricas y bioquímicas según su distribución, se realizaron por el test de Spearman $(\rho)$ o el test de Pearson ( $r$ ). Las diferencias significativas fueron consideradas con un valor de $\mathrm{p} \leq 0,05$. Los análisis se hicieron con el programa SPSS versión 22.

\section{Resultados}

La caracterización por sexo mostró similitud en edad, IMC y HOMA-IR. Fueron significativamente mayores en las niñas los valores de PGC, PC, ICC y la concentración de leptina, mientras que el índice cintura/cadera fue mayor en niños (tabla 1 ).

Las correlaciones por sexo de la leptina con las variables antropométricas fueron significativas en ambos sexos, con un grado de asociación mayor en las niñas para IMC, PGC PA y PC (tabla 2). El índice de cintura/ cadera tuvo correlación negativa pero no significante en ambos sexos. La asociación de la leptina con insulina y con el índice HOMA-IR fue significativa y moderada en ambos sexos (tabla 2).

Se exploraron además correlaciones de las variables antropométricas por edad según sexo, siendo significativa con talla y peso, pero en las niñas fue mayor el 
Tabla 1. Variables bioquímicas y antropométricas de niños prepúberes según sexo

\begin{tabular}{|c|c|c|c|c|}
\hline & \multicolumn{2}{|c|}{ Niños $(n=54)$} & \multicolumn{2}{|c|}{ Niñas $(n=56)$} \\
\hline & Promedio \pm DS & IC 95\% & Promedio \pm DS & IC 95\% \\
\hline Edad (años) & $7,8 \pm 1,3$ & $7,4-8,1$ & $7,9 \pm 1,2$ & $7,6-8,3$ \\
\hline Talla (cm) & $126 \pm 7$ & $124-128$ & $126 \pm 8$ & $124-128$ \\
\hline Peso $(k g)$ & $25,8 \pm 3,6$ & $24,8-26,7$ & $26,2 \pm 5,2$ & $24,8-27,6$ \\
\hline $\mathrm{IMC}\left(\mathrm{kg} / \mathrm{m}^{2}\right)$ & $16,1 \pm 1,2$ & $15,7-16,4$ & $16,4 \pm 1,8$ & $15,9-16,8$ \\
\hline PGC $(\%)^{* *}$ & $18,4 \pm 3,5$ & $17,5-19,3$ & $22,4 \pm 4,1$ & $21,3-23,5$ \\
\hline $\mathrm{PA}(\mathrm{cm})$ & $57,1 \pm 3,4$ & $56,2-58,1$ & $56,2 \pm 4,4$ & $55,1-57,4$ \\
\hline $\mathrm{PC}(\mathrm{cm})^{*}$ & $65,0 \pm 4,5$ & $63,8-66,2$ & $67 \pm 5,8$ & $65,8-68,9$ \\
\hline$I C C^{* *}$ & $0,88 \pm 0,04$ & $0,87-0,89$ & $0,84 \pm 0,04$ & $0,83-0,85$ \\
\hline Glicemia (mmol/L) & $4,9 \pm 0,3$ & $4,8-5,0$ & $4,8 \pm 0,4$ & $4,7-4,9$ \\
\hline Insulina $(\mu \cup / L)$ & $5,2 \pm 2,5$ & $4,6-6,0$ & $7,1 \pm 4,5$ & $5,9-8,3$ \\
\hline HOMA-IR & $1,2 \pm 0,6$ & $0,9-1,3$ & $1,5 \pm 1,0$ & $1,2-1,8$ \\
\hline Leptina $(\mathrm{ng} / \mathrm{ml})^{\star *}$ & $3,3 \pm 3,7$ & $2,3-4,3$ & $6,9 \pm 5,0$ & $5,5-8,2$ \\
\hline
\end{tabular}

El valor $p$ se calculó con el test de la U de Mann Whitney. * $p<0,05 * * p<0,01$; IMC: Índice de masa corporal, PGC: Porcentaje de grasa corporal; PA: Perímetro abdominal; PC: Perímetro de cadera, ICC: Índice cintura cadera.

Tabla 2. Correlaciones de las concentraciones de leptina, según sexo, con variables antropométricas y bioquímicas

\begin{tabular}{|c|c|c|c|}
\hline & $\begin{array}{c}\text { Total } \\
(\mathrm{n}=110)\end{array}$ & $\begin{array}{c}\text { Niños } \\
(n=54)\end{array}$ & $\begin{array}{c}\text { Niñas } \\
(n=56)\end{array}$ \\
\hline Edad (años) & $0,37 * *$ & 0,31 * & $0,44 * *$ \\
\hline Talla $(\mathrm{cm})$ & $0,35 * *$ & $0,41 * *$ & $0,42 * *$ \\
\hline Peso $(\mathrm{kg})$ & $0,54^{* *}$ & $0,52 * *$ & $0,69 * *$ \\
\hline IMC $\left(\mathrm{kg} / \mathrm{m}^{2}\right)$ & $0,56^{* *}$ & $0,35^{* *}$ & 0,72 ** \\
\hline PZ- IMC & $0,44^{* *}$ & $0,29 *$ & $0,56^{* *}$ \\
\hline PGC (\%) & $0,79 * *$ & 0,61 ** & 0,79 ** \\
\hline $\mathrm{PA}(\mathrm{cm})$ & $0,47^{* *}$ & $0,45^{* *}$ & $0,69 * *$ \\
\hline $\mathrm{PC}(\mathrm{cm})$ & $0,69 * *$ & $0,56^{* *}$ & $0,78^{* *}$ \\
\hline ICC & $-0,35$ ** & $-0,20$ & $-0,23$ \\
\hline Glicemia (mmol/L) & 0,05 & 0,13 & 0,10 \\
\hline Insulina (mU/L) & $0,65^{* *}$ & $0,62 * *$ & $0,65 * *$ \\
\hline HOMA-IR & $0,62 * *$ & $0,61 * *$ & $0,62 * *$ \\
\hline
\end{tabular}

Los coeficientes de correlación fueron calculados con el test de Spearman ( $\rho$ ); PA: Perímetro abdominal; PC: Perímetro de cadera; ICC: Índice cintura/cadera; Glicemia en ayuno. ${ }^{*} p<0,05$. $* * p<0,01$ grado de asociación del IMC con el PC $(\mathrm{r}=0,86 ; \mathrm{p}=0,000)$ y el PA $(r=0,82 ; p=0,000)$. La correlación del PGC con la edad solo fue significativa en las niñas (tabla 3 ). No hubo correlación significante entre la edad y el IMC en niños a diferencia de las niñas.
Tabla 3. Correlaciones de edad e IMC según sexo

\begin{tabular}{|c|c|c|c|c|}
\hline & \multicolumn{2}{|c|}{ Niñas $(n=56)$} & \multicolumn{2}{|c|}{ Niños $(n=54)$} \\
\hline & Edad & IMC & Edad & IMC \\
\hline Peso (kg) & 0,79 ** & & $0,73^{* *}$ & \\
\hline Talla (cm) & 0,87 ** & & $0,85^{\star *}$ & \\
\hline IMC $\left(\mathrm{kg} / \mathrm{m}^{2}\right)$ & $0,40 * *$ & & 0,14 & \\
\hline PGC (\%) & $0,43^{* *}$ & $0,79 * *$ & 0,24 & $0,45^{* *}$ \\
\hline $\mathrm{PA}(\mathrm{cm})$ & 0,51 ** & $0,86^{* *}$ & $0,45^{* *}$ & $0,65^{* *}$ \\
\hline $\mathrm{PC}(\mathrm{cm})$ & $0,72^{* *}$ & $0,82^{* *}$ & $0,65^{\star *}$ & $0,56^{* *}$ \\
\hline Insulina (mU/L) & 0,24 & 0,25 & 0,19 & $0,28^{*}$ \\
\hline Glicemia (mmol/L) & 0,39 ** & 0,15 & $0,28 *$ & 0,008 \\
\hline Leptina (ng/ml) & 0,41 ** & $0,65 * *$ & 0,25 & 0,39 ** \\
\hline HOMA & 0,28 & 0,24 & 0,20 & 0,26 \\
\hline
\end{tabular}

Los coeficientes de correlación fueron calculados con el test de Pearson. ${ }^{\star} p<0,05 .{ }^{*} p<0,001$. IMC: Índice de masa corporal; PGC: Porcentaje de grasa corporal; PA: Perímetro abdominal; PC: Perímetro de cadera.

\section{Discusión}

En este estudio se evaluó la relación de la concentración de leptina circulante con la composición corporal y la resistencia a la insulina en prepúberes normopeso según el sexo. 
Encontramos que entre los 6 a 10 años, a pesar de no tener diferencias en el IMC, las niñas presentaron valores mayores de PGC, PC, concentración de leptina e insulina y un menor índice de cintura/cadera que los niños.

La diferencia en adiposidad por sexo hallada, ha sido reportada en otros estudios con población similar, como el de Benjumea et al. ${ }^{22}$, quienes encontraron que después de los 6 años, la adiposidad se incrementa significativamente en ambos sexos, pero más en las niñas $(\mathrm{p}=0,000)$. Garnett et al. ${ }^{23}$, también reportaron en prepúberes entre los 7 a 8 años una mayor composición grasa en niñas. El dimorfismo sexual de la masa grasa en nuestro estudio, puede explicar la correlación significante entre la edad y el IMC solo en las niñas, hallazgo descrito desde edades más tempranas ${ }^{11,12,24}$.

La concentración de leptina circulante fueron significativamente mayores en niñas resultado similar al encontrado por Poveda et al. ${ }^{9}$ que reportaron concentraciones más altas de leptina en niñas normopeso. Si bien Thomsen et al. ${ }^{25}$ también obtuvieron resultados similares, es importante enfatizar que la población estudiada fue de 6 a 18 años y el análisis incluyó sujetos con y sin obesidad, a diferencia de nuestro estudio que solo incluyó niños normopeso de 6 a 10 años, y sin inflamación que puede alterar la concentración de leptina.

El hallazgo de un mayor PGC y su asociación con leptina significativa en niñas, podría explicarse por una tasa de adipogénesis aumentada ${ }^{3,23}$, probablemente por los niveles incipientes de estrógenos, del factor de crecimiento insulínico tipo I (IGF-I) ${ }^{23}$ y de otras hormonas como leptina e insulina ${ }^{3,8,26}$ en estas edades. Estos cambios prepuberales podrían estar anticipando el desarrollo de la etapa puberal, en la que la leptina como indicador de reservas de energía en las niñas, se ha vinculado con la preparación para la reproducción y la lactancia, mientras que en los niños tal reserva energética puede ser menor para garantizar la espermatogénesis ${ }^{27,28}$.

Teniendo en cuenta estos resultados según el sexo, las diferencias encontradas no podrían ser explicadas por el grado de RI, medida con el índice HOMA-IR, ni por la insulinemia, factores que en obesidad del adulto se han asociado a resistencia a la leptina e hiperleptine$\mathrm{mia}^{29}$. En modelos animales normopeso se ha descrito que los niveles elevados de leptina en presencia de alto PGC en las hembras, podría explicarse por un menor transporte a través de la membrana hematoencefáli$\mathrm{Ca}^{8,29,30}$, esto puede estar relacionado con una mayor concentración de leptina circulante en las niñas prepúberes que estimule la adipogénesis, sin cambios en los efectos centrales de la leptina, como la disminución de ingesta y el incremento del gasto calórico, todo lo cual contribuiría a la mayor PGC encontrada en las niñas.
Desde la etapa prepuberal los niveles de esteroides sexuales como el $17 \beta$-estradiol y la estrona son mayores en las niñas ${ }^{31}$. Se ha reportado in vitro que los estrógenos inducen una mayor secreción de leptina en adipocitos del sexo femenino. La mayor concentración de estrógenos y la alta sensibilidad a estas hormonas podría favorecer mayores niveles de leptina en las niñas ${ }^{32}$. No se descarta que otros mecanismos como las diferencias por sexo en la depuración, el transporte, la sensibilidad periférica y la autorregulación autocrina de la leptina, puedan estar relacionados con su dimorfismo sexual, procesos que ameritan ser profundizados en futuras investigaciones.

Las fortalezas de este estudio fueron el diagnóstico del estadio de Tanner por examen médico, la distribución homogénea por sexo y edad de la muestra y además que solo se incluyeron sujetos evaluados sin inflamación sistémica. Estas condiciones favorecen la confiabilidad de los resultados obtenidos. Una debilidad del estudio fue que no se realizaron mediciones de factores reguladores de la adipogénesis y secreción de la leptina, que pudieran ayudar a explicar los resultados asociados con su dimorfismo sexual.

\section{Conclusiones}

En prepúberes normopeso entre 6 a 10 años, se presentan diferencias por sexo en adiposidad y en los niveles de leptina, las cuales no parecen estar asociadas con diferencias en el IMC ni en la RI.

El alto grado de asociación de la leptina circulante con la adiposidad en las niñas, podría estar relacionada con una mayor tasa de adipogénesis inducida por esta hormona.

La mayor adiposidad en las niñas podría hacerla más susceptible a la obesidad en la etapa puberal o en la adultez, por lo cual es importante enfatizar en este grupo la adquisición de estilos de vida saludable desde una edad temprana.

\section{Responsabilidades Éticas}

Protección de personas y animales: Los autores declaran que los procedimientos seguidos se conformaron a las normas éticas del comité de experimentación humana responsable y de acuerdo con la Asociación Médica Mundial y la Declaración de Helsinki.

Confidencialidad de los datos: Los autores declaran que han seguido los protocolos de su centro de trabajo sobre la publicación de datos de pacientes.

Derecho a la privacidad y consentimiento informado: Los autores han obtenido el consentimiento in- 
formado de los pacientes y/o sujetos referidos en el artículo. Este documento obra en poder del autor de correspondencia.

\section{Conflicto de intereses}

Los autores declaran no tener conflicto de intereses.

\section{Referencias}

1. Rolland-Cachera MF, Deheeger M, Maillot M, Bellisle F. Early adiposity rebound: Causes and consequences for obesity in children and adults. Int J Obes. 28 de diciembre de 2006;30(S4):S11-7.

2. Dietz WH. Critical periods in childhood for the development of obesity. Am J Clin Nutr. 1994;59(5):955-9.

3. Lee M-J. Hormonal Regulation of Adipogenesis. Compr Physiol. 12 de septiembre de 2017;7(4):1151-95.

4. Acevedo M, Arnáiz P, Barja S, et al. Proteína $C$ reactiva y su relación con adiposidad, factores de riesgo cardiovascular y aterosclerosis subclínica en niños sanos. Elsevier. 2007;60(10):1051-8.

5. Rolland-Cachera MF, Deheeger M, Bellisle F, Sempé M, Guilloud-Bataille M, Patois E. Adiposity rebound in children: a simple indicator for predicting obesity. Am J Clin Nutr. 1984;39(1):129-35.

6. Arner E, Westermark PO, Spalding KL, et al. Adipocyte turnover: Relevance to human adipose tissue morphology. Diabetes. 2010;59(1):105-9.

7. Acosta M, Ramos F, Escobar J, et al. Mecanismos bioquímicos de la leptina implicados en el desarrollo de la obesidad. Rev Médica la Univ Veracruzana. 2015;15(2):103-13.

8. Clegg DJ, Riedy CA, Smith KAB, Benoit SC, Woods SC. Differential Sensitivity to Central Leptin and Insulin in Male and Female Rats. Am Diabetes Assoc. 2003;52(3):682-7.

9. Poveda E, Callas NE, Baracaldo CM, Castillo C, Hernández P. Concentración sérica de leptina en población escolar de cinco departamentos del centro-oriente colombiano y su relación con parámetros antropométricos y perfil lipídico. Biomédica. 2007;27:505-14.

10. Zhang M, Cheng H, Zhao X, et al. Leptin and Leptin-to-Adiponectin Ratio Predict Adiposity Gain in Nonobese Children over a Six-Year Period. Child Obes. 2017;13(3):213-21.

11. Fomon SJ, Nelson SE. Body composition of the male and female reference infants. Annu Rev Nutr. 2002;22(1):1-17.
12. Hawkes CP, Hourihane JO, Kenny LC, Irvine AD, Kiely M, Murray DM. Gender- and gestational age-specific body fat percentage at birth. Pediatrics. 2011;128(3):e645-51.

13. Onis M. WHO Child Growth Standards based on length/height, weight and age. Acta Paediatr (Oslo, Norw 1992) Suppl. 2006;450:76-85.

14. Marquant E, Plotton I, Reynaud R. Pubertad normal. EMC - Pediatría. 2015;50(1):1-6.

15. Gaete X, García R, Riquelme J, Codner E. La pubertad en niños chilenos muestra un adelantamiento en el inicio del crecimiento testicular. Rev Med Chile 2015; 143:297-303.

16. Silva VS da, Vieira MFS. International Society for the Advancement of Kinanthropometry (ISAK) Global: international accreditation scheme of the competent anthropometrist. Rev Bras Cineantropometria Desempenho Hum. 2020;22.

17. Ribeiro G dos S, Fragoso EB, Nunes $\mathrm{RD}$, Lopes AL. Erro técnico de medida em antropometria: análise de precisão e exatidão em diferentes plicômetros. Rev Educ Física/J Phys Educ. 2019;88(2):8107.

18. Slaughter MH, Lohman TG, Boileau RA, et al. Skinfold equations for estimations of body fatness in children and youth. Hum Biol. 1988;60(5):709-23.

19. Masuccio F, Verrotti A, Chiavaroli V, et al. Weight gain and insulin resistance in children treated with valproate: The influence of time. J Child Neurol. 2010;25(8):941-7.

20. Jensen AN, López M, Mir C, Martínez M, Pianese M, Erhard M. Relationship between serum leptin levels and sodium excretion in a local population of obese children and adolescents. RAEM. 2011;48(3):127-35.

21. Matthews DR, Hosker JR, Rudenski AS, Naylor BA, Treacher DF, Turner RC. Homeostasis model assessment: insulin resistance and fl-cell function from fasting plasma glucose and insulin concentrations in man. Diabetologia. 1985;28(7):412-9.

22. Benjumea-Rincón MV, Parra-Sánchez JH,
Ocampo-Téllez PR. Concordancia en la talla para la edad entre referencias NCHS y OMS en indígenas colombianos. Rev Salud Publica. 2016;18(4):503-15.

23. Garnett SP, Högler W, Blades B, et al. Relation between hormones and body composition, including bone, in prepubertal children. Am J Clin Nutr. 1 de octubre de 2004;80(4):966-72.

24. Pietrobelli A, Malavolti M, Fuiano N, Faith MS. The invisible fat. Acta Paediatr Int J Paediatr. 2007;96(Suppl. 454):35-8.

25. Lausten-Thomsen U, Lund MAV, Frithioff-Bøjsøe C, et al. Reference values for leptin/adiponectin ratio in healthy children and adolescents. Clin Chim Acta. 1 de junio de 2019;493:123-8.

26. Martos-Moreno GÁ, Barrios V, Chowen JA, Argente J. Adipokines in Childhood Obesity. En: Vitamins and Hormones. Academic Press Inc.; 2013. p. 107-42.

27. Sharrock KCB, Kuzawa CW Leonard WR, et al. Developmental changes in the relationship between leptin and adiposity among Tsimané children and adolescents. Am J Hum Biol. 2008;20(4):392-8.

28. Palmer BF, Clegg DJ. The sexual dimorphism of obesity. Mol Cell Endocrinol. 2015;402:113-9.

29. Munhoz R, Campos S, Cristina D, et al. Effects of magnitude of visceral adipose tissue reduction: Impact on insulin resistance, hyperleptinemia and cardiometabolic risk in adolescents with obesity after long-term weightloss therapy. Diabetes Vasc Dis Res. 2019;16(2):196-206.

30. Barateiro A, Mahú I, Domingos AI. Leptin Resistance and the NeuroAdipose Connection. Front Endocrinol (Lausanne). 2017;8:45.

31. Courant F, Aksglaede L, Antignac J, et al. Assessment of circulating sex steroid levels in prepubertal and pubertal boys and girls by a novel ultrasensitive gas chromatography-tandem mass spectrometry. J Clin Endocrinol Metab. 2010;95(1):82-92.

32. Roemmich JN, Rogol AD. Role of leptin during childhood growth and development. Endocrinol Metab Clin North Am. 1999;28(4):749-64. 